\section{Intensivierte Therapie bei Hochrisiko-DLBCL?}

In der vorliegenden Studie wurde bei Patienten mit primärem diffus-großzelligem B-Zell-Lymphom (DLBCL) und hohem klinischen Risiko eine intensivierte Chemoimmuntherapie mit zusätzlicher ZNS-Prophylaxe untersucht.

$\mathrm{D}$ ie konventionelle CHOP-Chemotherapie in Kombination mit dem monoklonalen CD20-Antikörper Rituximab (R-CHOP) ist die Standardbehandlung beim DLBCL. In einer Phase-II-Studie der Nordic Lymphoma Group wurde eine intensivierte Chemoimmuntherapie mit einer systemischen ZNS-Prophylaxe bei diesen Patienten untersucht.

156 Patienten im Alter von 20-64 Jahren (Median: 54 Jahre) wurden in die Studie aufgenommen. Alle hatten ein primäres DLBCL oder ein follikuläres Lymphom vom Grad III ohne klinische Zeichen einer ZNS-Erkrankung, eine negative Liquor-Zytologie, einen altersadjustierten internationalen PrognoseIndex von 2-3 und einen WHO-Performance-Score von 0-3.
Die Therapie bestand aus sechs Zyklen R-CHOEP-14 (Rituximab plus Cyclophosphamid, Doxorubicin, Vincristin, Etoposid und Prednison alle zwei Wochen). Zur ZNS-Prophylaxe erhielten die Patienten anschließend je einen Zyklus hoch dosiertes Cytarabin bzw. Methotrexat. $17 \%$ der Patienten erhielten zusätzlich eine Radiotherapie. Der primäre Endpunkt war das Überleben ohne Therapieversagen nach drei Jahren.

Das 3-Jahres-Gesamtüberleben betrug $81 \%$, das 3-Jahres-Überleben ohne Therapieversagen $65 \%$. Patienten mit hohem Ki67-Score hatten ein signifikant besseres Überleben ohne Therapieversagen als Patienten mit niedrigem Ki67Score. Bei sieben Patienten kam es innerhalb von sechs Monaten zu einem ZNS-
Rezidiv. Diese Rate war niedriger als erwartet.

Gleichzeitig war die toxizitätsbedingte Sterberate mit drei Todesfällen relativ niedrig. Die am häufigsten beobachteten toxischen Effekte waren hämatologischer Natur (14\% vom Grad 3 bzw. $79 \%$ vom Grad 4). Weitere Toxizitäten der Grade 3-4 waren Mukositis, gastrointestinale Ereignisse und Infektionen.

Fazit: Den Autoren zufolge brachte die intensivierte Therapie gute Ergebnisse hinsichtlich des Gesamtüberlebens und des Überlebens ohne Therapieversagen. Damit scheint es möglich zu sein, das Auftreten einer ZNS-Progression durch eine frühe ZNS-Prophylaxe zu reduzieren.

Judith Neumaier

Holte $\mathrm{H}$ et al. Dose-densified chemoimmunotherapy followed by systemic central nervous system prophylaxis for younger high-risk diffuse large B-cell/follicular grade 3 lymphoma patients: Results of a phase II Nordic Lymphoma Group study. Ann Oncol. 2012 Dec 17. [Epub ahead of print].

\title{
Primäres ZNS-Lymphom: Gute Langzeit-Daten
}

\section{Die Hochdosis-Chemotherapie mit anschließender autologer Stammzelltrans- plantation ist ein vielversprechender Ansatz für ausgewählte Patienten mit primärem ZNS-Lymphom. Wie sehen die Langzeitergebnisse aus?}

$\mathrm{n}$ zwei Studien mit insgesamt 43 unter 67 Jahre alten immunkompetenten $\mathrm{Pa}$ tienten mit neu diagnostiziertem primärem ZNS-Lymphom (PCNSL) wurden Überleben und Rezidivraten über median 120 Monate untersucht. Die Behandlung erfolgte mit einer Hochdosistherapie, die aus einer Induktionstherapie mit drei bzw. vier Zyklen Methotrexat (8 g/ $\mathrm{m}^{2}$ i.v.) und einer Konditionierungsphase mit Carmustin $\left(400 \mathrm{mg} / \mathrm{m}^{2}\right)$ und Thiothepa $(2 \times 5 \mathrm{mg} / \mathrm{kg}$ bzw. $4 \times 5 \mathrm{mg} /$ $\mathrm{kg}$ ) bestand. In beiden Studien folgte dann eine autologe Stammzelltransplantation. Eine Ganzhirnbestrahlung fand in der ersten Studie bei allen, in der zweiten Studie nur bei einem Teil der Patienten statt.

In einer gepoolten Analyse wurden die Langzeitdaten der beiden Studien zusammen ausgewertet. 34 Patienten er- reichten eine vollständige Remission. Zwölf (35\%) von ihnen hatten später ein Rezidiv. In der Per-Protokoll-Population war die Rezidivrate mit $30 \%$ niedriger.

Das mediane Gesamtüberleben betrug 104 Monate. Das 2- und 5-JahresGesamtüberleben betrug $81 \%$ bzw. $70 \%$, das 2- und 5-Jahres-Überleben $81 \%$ bzw. $67 \%$. In der Per-Protocol-Analysis $(\mathrm{n}=$ 34) betrug das 5-Jahres-Gesamtüberleben $82 \%$ und das ereignisfreie 5-JahresÜberleben $79 \% .28$ Patienten lebten länger als fünf Jahre. Bei sechs von ihnen kam es nach mehr als fünf Jahren zu einem Rezidiv. Nur bei zwei davon war die Salvage-Therapie erfolgreich, sodass sie noch leben.

Insgesamt acht Patienten (9\%) litten unter Neurotoxizität, alle hatten eine Ganzhirnbestrahlung bekommen. Diesbezüglich wurde in der zweiten Studie das Behandlungsprotokoll verbessert: Die Ganzhirnbestrahlung erfolgte nur bei den Patienten, die nicht vollständig auf die Chemotherapie, die in dieser Studie höher dosiert war als in der ersten, ansprachen. Dieses Vorgehen reduzierte die Häufigkeit der Neurotoxizität deutlich, es war nur eine Patientin betroffen. Auf die Wirksamkeit hatte das Fehlen der Ganzhirnbestrahlung offensichtlich keinen Einfluss, denn die zehn Patienten, die keine Bestrahlung erhielten, erreichten eine Ansprechrate von $100 \%$. Das 2und 5-Jahres-Gesamtüberleben betrug 80 bzw. $70 \%$.

Fazit: Die in dieser Studie verwendete Hochdosis-Chemotherapie mit anschlieBender Stammzelltransplantation erwies sich als sicher und führte zu hohen Überlebensraten bei ausgewählten Patienten mit neu diagnostiziertem primärem ZNS-Lymphom.

Judith Neumaier

Kasenda B et al. Prognosis after high-dose chemotherapy followed by autologous stem-cell transplantation as first-line treatment in primary CNS lymphoma - a long-term follow-up study. Ann Oncol. 2012;23(10):2670-5. 